\title{
Regeneration following tibial nerve crush in the rabbit: the effect of proximal constriction
}

\author{
K REINERS, R W GILLIATT, A E HARDING, J H O'NEILL \\ From the University Department of Clinical Neurology, Institute of Neurology, Queen Square, London, UK
}

SUMMARY In order to test the regenerative capacity of atrophic axons, a constricting ligature was placed around the proximal tibial nerve of the rabbit, and the nerve crushed at the ankle one week later. Axonal atrophy with altered $\mathrm{g}$ ratios was subsequently confirmed in fibres distal to the site of ligature and proximal to the site of crush. In nerves with tight proximal ligatures the reinnervation of plantar muscles and the subsequent recovery of distal motor latency were delayed, indicating impaired regeneration. This result may be relevant to the "double-crush" theory of nerve damage.

A constricting ligature round the proximal tibial nerve in the rabbit is known to cause a reduction in distal axonal calibre. This only occurs if the ligature is tight enough to produce Wallerian degeneration in a substantial proportion of the large myelinated fibres. The surviving fibres then develop distal axonal atrophy, as shown in teased fibres by a decrease in external fibre diameter relative to internodal length, and in transverse sections by a decrease in the ratio of axon diameter to external fibre diameter (g ratio). Paranodal demyelination and fibre degeneration commencing distally may be seen in severely affected nerves. ${ }^{1}$ These morphological changes are accompanied by a reduction in maximal motor conduction velocity distal to the ligature. ${ }^{2}$

The present experiments, also using rabbits, were designed to examine the regenerative capacity of atrophic nerve fibres distal to a constriction. A ligature was placed round the tibial nerve in the thigh, followed one week later by nerve crush just above the ankle. The time required for reinnervation of the plantar muscles was assessed by tibial nerve stimulation at the ankle, and compared with the time required after crush alone. Once reinnervation had occurred, the gradual recovery of distal motor latency was followed by serial nerve conduction studies.

\section{Methods}

The experiments were performed on male New Zealand white rabbits aged 3-5 months. Under general anaesthesia

Address for reprint requests: Professor $\mathbf{R}$ W Gilliatt, University Department of Clinical Neurology, Institute of Neurology, Queen Square, London WCIN 3BG, UK.

Received 4 March 1986.

Accepted 23 May 1986 (intramuscular ketamine and xylazine), and with an aseptic technique, a silk ligature (Ethicon braided $0.2 \mathrm{~mm}$ gauge) was tied round the tibial branch of the sciatic nerve in the lower thigh on one side and left in place. The ligatures were tied tightly enough to produce Wallerian degeneration of more than $50 \%$ of the large motor fibres, as estimated by the reduction in amplitude of the muscle action potential evoked by nerve stimulation at the ankle one week after ligature. ${ }^{1}$ In a second group of animals similar ligatures were applied but removed after 24 hours; the amount of Wallerian degeneration caused by the ligature was again checked by distal nerve stimulation and muscle recording after one week. Immediately after nerve stimulation, and under the same anaesthetic, the tibial nerve was crushed with smooth-tipped forceps $1 \mathrm{~cm}$ above the ankle. The operation was performed with an aseptic technique, the procedure being the same for animals with persistent ligatures and those with ligatures released. In a control group of animals the tibial nerve was crushed at the same level without a preceding proximal ligature.

One to two weeks after nerve crush animals were reexamined under anaesthesia to ensure that complete degeneration of motor fibres to the plantar muscles had occurred. Starting three weeks after crush, nerve stimulation was carried out at intervals of 3-4 days in order to detect the first evidence of reinnervation of the plantar muscles. Once this had occurred, conduction studies were repeated at weekly intervals under light anaesthesia, to follow the time-course of recovery of distal latency and muscle action potential (MAP) wave-form and amplitude.

For nerve stimulation a needle electrode inserted close to the tibial nerve trunk at the ankle, just distal to the site of crush, was used as the stimulating cathode, and a remote electrode placed subcutaneously near the knee. A stimulus intensity several times greater than that necessary to stimulate a normal nerve was used when testing for early regeneration. Muscle action potentials from the plantar muscles were recorded through subcutaneous electrodes as described previously. ${ }^{1}$ The distance from the proximal ligature to the site of crush varied from 10.8 to $12.9 \mathrm{~cm}$, distal stimulation 
Table 1 Times of reinnervation of the plantar muscles after nerve crush above the ankle

\begin{tabular}{llll}
\hline & & \multicolumn{1}{l}{ First detectable reinnervation (days after crush) } \\
\cline { 2 - 4 } & No of nerves & Median & Range \\
\hline A Crush alone & 11 & 34 & $31-38$ \\
B Persistent ligature & 12 & 37 & $31-48$ \\
B1* & 6 & 34 & $31-38$ \\
B2 $\dagger$ & 6 & 41 & $35-48$ \\
C Ligature removed & 8 & 35 & $28-39$ \\
& A vs B: $p<0.05$ & & \\
& B1 vs B2: $p<0 \cdot 01$ & & \\
\hline
\end{tabular}

*55-68\% reduction in MAP amplitude after ligature.

$+85-95 \%$ reduction in MAP amplitude after ligature.

being carried out approximately $1.0 \mathrm{~cm}$ below the site of crush. Conduction distance from the stimulating cathode at the ankle to the active recording electrode in the foot varied from 6 to $7 \mathrm{~cm}$.

Animals were killed with iv pentobarbitone 10-12 weeks after nerve crush. Removal of the tibial and medial plantar nerves, their preparation for teasing and transverse sections, and examination and measurement were performed as in earlier studies. ${ }^{1}$

\section{Physiological results}

The time after nerve crush at which reinnervation of

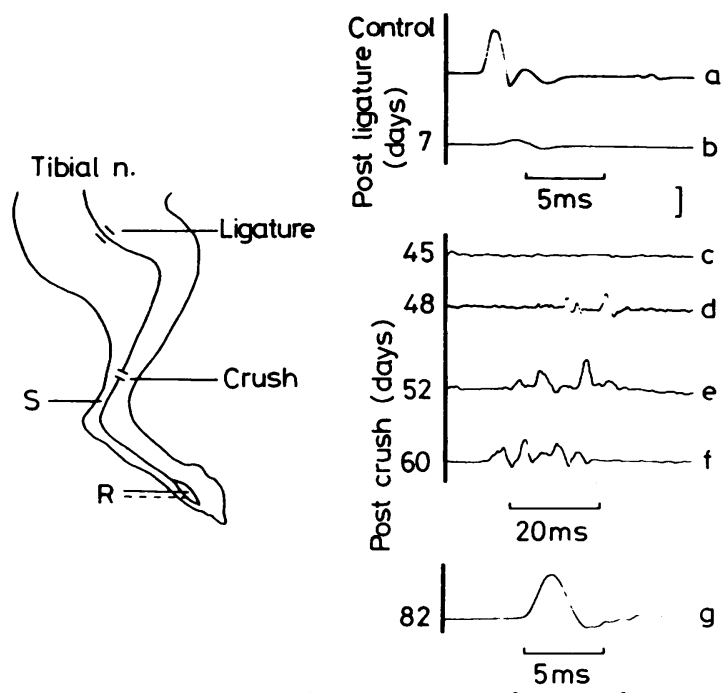

Fig 1 (Left) Diagram showing position of proximal ligature, distal crush, stimulating $(S)$ and recording $(R)$ electrodes. (Right) Plantar muscle action potentials evoked by tibial nerve stimulation (a) before, and (b) 7 days after the application of a proximal ligature, and $((c)-(g))$ at intervals after distal nerve crush, which was performed immediately after recording (b). Evidence of reinnervation can be seen in recording (d) but not in (c). Vertical bar represents $5 \mathrm{mV}$ in $(a), 2 \mathrm{mV}$ in (b) and $(\mathrm{g}), 0.1 \mathrm{mV}$ in (c), (d) and (e), and $0.5 \mathrm{mV}$ in $(f)$. the plantar muscles was first detected is shown in table 1 . Since the nerve crush was carried out at a standard level in animals which were similar in age and weight, results in the different groups can be directly compared. In eleven unconstricted nerves used as controls (group A) reinnervation times ranged from 31-38 days with a median of 34 days. In the twelve nerves in which a proximal ligature had been left in place (group B) there was a wide scatter, some values being within the normal range but others being increased up to 48 days. The difference between groups A and B was significant at the $5 \%$ level. In contrast, the reinnervation times for nerves in which proximal ligatures had been released after 24 hours (group C) were similar to those of controls.

A possible cause for the variation between different nerves within group B was thought to be the tightness of the ligature in individual cases. Previous experience had shown that some indication of the severity of constriction of surviving fibres could be obtained from the amount of Wallerian degeneration caused by the ligature, as indicated by the reduction in MAP amplitude following its application. ${ }^{1}$ In the group B nerves of the present series, there were six in which the post-operative reduction in MAP amplitude ranged from $55 \%-68 \%$ (group B1) and six in which there was a more marked reduction ranging from $85 \%-95 \%$ (group B2). Reinnervation times for the two sub-groups are shown in table 1 , and it is clear that it was only in group B2 that there was delayed reinnervation compared with controls. Six of the nerves in group $\mathrm{C}$ (ligatures released) had also shown marked reductions in post-operative MAP amplitude $(84 \%-98 \%)$, but when their reinnervation times were analysed separately, they did not differ significantly from those of controls.

Immediately after reinnervation, evoked MAPs were of low voltage and their latency was increased approximately tenfold. As more muscle fibres were reinnervated, the evoked MAPs showed temporal dispersion. Subsequently distal latency fell and MAP 


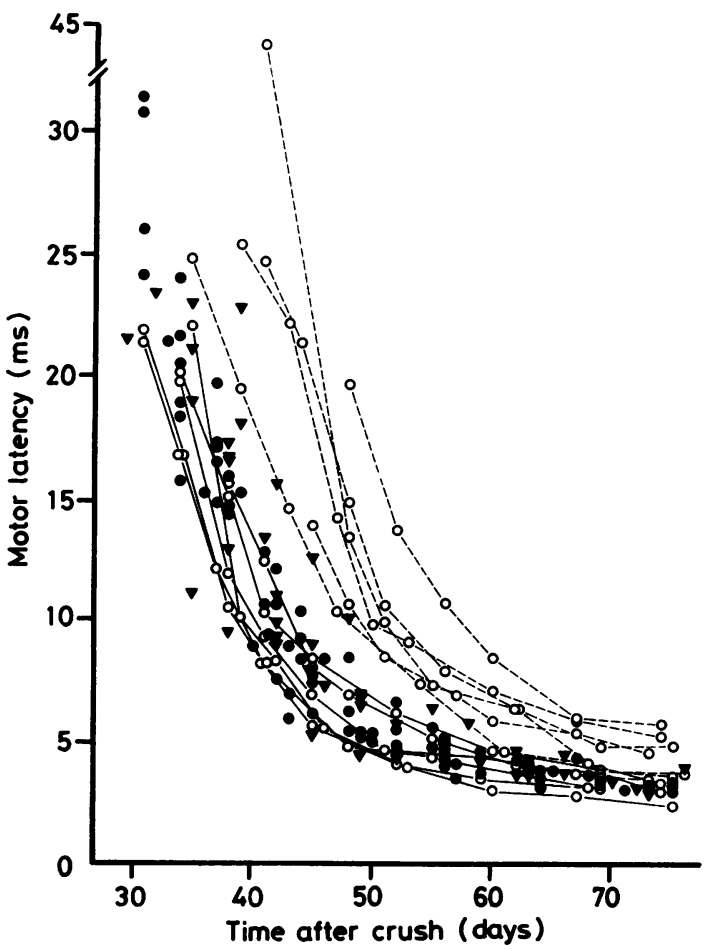

Fig 2 Latency of plantar muscle action potentials evoked by stimulating tibial nerve after crush. Filled circles indicate values for group $A$ (crush alone), open circles group $B$ (persistent ligature), filled triangles group $C$ (ligature removed). Values for individual group $B$ animals are joined by a continuous line (BI) or a broken line (B2).

wave-form gradually returned to normal. An illustrative example from an animal in group B2 is shown in fig 1. Evoked MAP amplitude, initially $7 \mathrm{mV}$, had fallen to $0.5 \mathrm{mV}$ within one week of ligature, indicating a severe constriction accompanied by Wallerian degeneration of most of the motor fibres. The surviving fibres were then crushed above the ankle, and 48 days elapsed before small evoked MAPs (amplitude less than $50 \mu \mathrm{V}$ ) were seen, the latency of the earliest component being $20 \mathrm{~ms}$. In subsequent recordings (not all of which are shown in fig 1 ) distal latency gradually fell to a final figure of $5.8 \mathrm{~ms}$ at 82 days, by which time MAP wave-form was normal, although the duration of the negative deflection was increased compared with the potential recorded one week after ligature and immediately before nerve crush. It can also be seen that MAP amplitude, which was $0.5 \mathrm{mV}$ immediately before crush, had increased to $3 \mathrm{mV}$ by 82 days. This was presumably the result of axon branching during regeneration, with the incorporation of increasing numbers of denervated muscle fibres into surviving motor units. No other animal in group B2 showed such a large increase in MAP amplitude after regeneration. In the remainder of group B2 the increase was less than with lesions of comparable severity in group $\mathbf{C}$, although individual variation and the small numbers make the results unsuitable for statistical evaluation.

Figure 2 shows MAP latencies for group A, B and $C$ animals at different intervals after crush. Values immediately after reinnervation were mostly in the range $20-30 \mathrm{~ms}$ (compared with a pre-operative range of $1.5-2.5 \mathrm{~ms}$ ) but in the next few weeks there was a relatively rapid fall which was succeeded by a slower rate of decline. For group B the points have been joined to show the recovery of individual animals; the six animals in group B2 (broken lines) can be seen on the right. In all six motor latency was slow to fall compared with the remaining animals, and at 70-80 days after crush the latency was still prolonged in four.

It should be added that although the tying of the ligature in the thigh caused Wallerian degeneration in most of the motor fibres to the plantar muscles, the nature of the lesion and the distance from the ligature to the muscles $(18-21 \mathrm{~cm})$ were such that regeneration of these fibres did not result in muscle reinnervation within the experimental period. The results shown in fig 2 were solely due to the regeneration of fibres which had survived the ligature and which were then crushed near the ankle.

\section{Histological results}

Transverse sections of the tibial nerve in the lower thigh were cut with the constricting ligatures in place in 11 of the 12 animals in group B. Skip serial sections were used to establish the level at which constriction

Table 2 Cross sectional area of tibial nerves at the site of ligature compared to unconstricted nerve area at the same level

\begin{tabular}{llll}
\hline & & \multicolumn{2}{l}{ Cross sectional area $\left(\mathrm{mm}^{2}\right)$} \\
\cline { 3 - 4 } & No of nerves & Mean & Range \\
\hline A Crush alone & 4 & 0.81 & $0.76-0.93$ \\
B Persistent ligature & 6 & 0.56 & $0.48-0.79$ \\
B2 & 5 & 0.46 & $0.41-0.52$ \\
\hline
\end{tabular}


Table 3 Axon/external diameter ( $g$ ) ratios for tibial nerve fibres with external diameters between 7 and $11 \mu \mathrm{m}$

$\left.\begin{array}{lccl}\hline & \text { No of fibres } & \text { No of animals } & \text { g ratio (mean } \pm S D \text { ) } \\ \hline \text { Unoperated control nerves } & 3245 & 21 & 0.61 \pm 0.04 \\ \text { A Crush alone } & 693 & 4 & 0.56 \pm 0.05 \\ \text { C Ligature removed } & 307 & 5 & 0.58 \pm 0.04 \\ \text { B Persistent ligature } & 288 & 6 & 0.51 \pm 0.05 \\ \text { B1 } & 267 & 6 & 0.48 \pm 0.06\end{array}\right\} \dagger$

$* t=17 \cdot 7, p<0.001 . \quad t t=6.37, p<0.001$

was maximal. The area within the ligature was then measured for comparison with the total fascicular area of group A nerves in transverse sections taken at the same level in the thigh (table 2). Group A nerves were used as controls rather than normal nerves, since it was thought possible that a distal crush might have a retrograde effect on fascicular area. The ligatures in group B nerves caused a reduction in cross-sectional area of approximately $40 \%$, the reduction being slightly greater in group B2 than in group B1.

Transverse sections of the tibial nerves in the leg proximal to the site of crush confirmed the presence of extensive Wallerian degeneration in both group B and group $C$ nerves. In some cases the changes were severe, and few large myelinated fibres were available for measurement of axon and external fibre diameter. However, by pooling results from different animals, sufficient numbers were obtained for statistical analysis.

In table 3 axon/external diameter ratios (g ratios) for the large myelinated fibres $(7-11 \mu \mathrm{m}$ external diameter) of groups A, B and C nerves are compared with those of normal nerves taken from the unoperated legs of the same animals, examined at the same level. When compared with the normal unoperated nerves, group A nerves showed a small reduction in mean $\mathrm{g}$ ratio, of approximately $8 \%$, proximal to crush alone. In group $B$ the mean $\mathrm{g}$ ratio was reduced by $19 \%$, the reduction being slightly greater in group B2 than in group B1. In group C the reduction in mean $g$ ratio was no greater than after crush alone.

When individual teased fibres from the tibial nerve in the leg proximal to the site of crush were examined, the changes in the ligatured nerves were similar to those noted in previous studies, namely thinning of the fibres with irregularity of outline and occasional paranodal demyelination. These changes were less marked in group $C$ nerves (proximal ligature released) than in group B (ligature maintained), but in some nerves from both groups the number of surviving fibres was too small to allow useful statistical comparison.

In transverse sections of the plantar nerves in the foot, $g$ ratios for the regenerated fibres were not significantly different in groups A, B and C (table 4). Teased fibres from the plantar nerves had uniformly short internodes, typical of regeneration, but no other abnormality was noted. In particular, there was no irregularity of the internodal myelin and no paranodal changes were seen.

Two additional animals were killed 15 and 18 days after a proximal ligature had been applied to the tibial nerve, and 7 and 10 days after distal crush. The fall in evoked MAP amplitude one week after ligature was $52 \%$ and $91 \%$ respectively. Examination of loosely teased fibres immediately proximal to the site of crush showed widening of one, or occasionally two, nodes of Ranvier in less than $10 \%$ of fibres. There was no evidence of more extensive "dying back" from the level of the crush, as previously described by MorganHughes $e a^{3}$ in nerves from animals with acrylamide neuropathy.

\section{Discussion}

Delayed reinnervation of distal muscles is known to occur when regenerating fibres pass through a constricted region. ${ }^{4}$ This experimental situation has now been reversed, with the constricting ligature proximal to the site of crush, and delayed reinnervation and/or maturation was observed in six out of the 12 animals

Table 4 Axon/external diameter $(g)$ ratios for medial plantar nerve fibres with external diameters between 3 and $7 \mu m$

\begin{tabular}{lllll}
\hline & No of fibres & No of animals & $\mathrm{g}$ ratio (mean \pm SD) \\
\hline A Crush alone & 598 & 2 & $0.67 \pm 0.05$ \\
B Persistent ligature & 672 & $2 \dagger$ & $0.65 \pm 0.05$ \\
C Ligature removed & 631 & 2 & $0.66 \pm 0.05$ & 0 \\
\hline
\end{tabular}

$* t=1.59, p>0 \cdot 1$.

+Both animals from group B2. 
studied. In the previous experiments it seemed likely that part of the delay occurred when regenerating fibres attempted to penetrate the constricted region; indeed only a proportion of them succeeded in doing so. In the present study it is reasonable to relate the delay to the atrophic changes in the axons themselves. There is support for this in the fact that tighter constrictions, which had produced more marked distal axonal atrophy, were the ones which also produced delayed reinnervation. Since the constricting ligatures had to be tied tightly enough to produce immediate Wallerian degeneration of most of the large myelinated fibres, it was important to establish that the degeneration itself did not influence reinnervation by surviving fibres. Accordingly a series of animals was studied in which the ligatures were removed after 24 hours; in this group muscle reinnervation after distal crush was not delayed.

The mechanism of delayed reinnervation and maturation by atrophic axons is of considerable interest. It is known that perikaryal synthesis of neurofilament protein is reduced after axotomy. ${ }^{5}$ In the present experiments, could some abnormal signal transported centripetally from the site of constriction trigger a decrease in perikaryal neurofilament protein synthesis, which might then be responsible both for the distal axonal atrophy and for the delayed regeneration? This would seem to be excluded by the known transport rate of neurofilament protein along the axon, which is so slow that it could not influence events in the distal axon in the time available. It is possible that fast transport systems, such as the delivery of membrane components required for incorporation into regenerating sprouts, are abnormal in this model.

Toxic agents which impair regeneration may have a much greater effect than that seen here. For example, severely delayed regeneration has recently been described in animals given small doses of vincristine. ${ }^{6}$ With acrylamide there is actual dying back from the site of crush as well as impaired regeneration; ${ }^{37}$ this was not observed in the present study.

The increase in evoked MAP amplitude which we observed after reinnervation suggests that surviving axons distal to the constriction were capable of branching during regeneration, and of reinnervating muscle fibres which had been denervated when the ligature was tied. The numbers in the present experiments were too small to establish whether the branching was as effective in these cases as after ligature release, and this particular aspect requires further study.

In 1973 Upton and $\mathrm{McComas}^{\circ}$ put forward the "double-crush" hypothesis, proposing that a proximal compressive or constrictive lesion would render the same axons more vulnerable to mechanical dam- age further distally, by an effect on proximo-distal axonal transport. While our results indicate that delayed regeneration can occur in a "double-crush" situation, it is clear that it only occurs under strictly circumscribed conditions. It did not occur, for example, in a previous study of regeneration distal to an acute demyelinating block without continuing constriction. ${ }^{9}$ In order to demonstrate the phenomenon in the present experiments it was necessary for the proximal constriction to be so severe that the majority of myelinated fibres degenerated at that level, with a significant reduction in the distal axonal calibre of surviving fibres. Upton and McComas' original hypothesis was based on the clinical association of median and ulnar entrapment with cervical spondylosis. ${ }^{8}$ More recent work suggests that the common factor in their cases was likely to have been osteoarthritis affecting the spine as well as the limb joints, rather than a "double-crush" mechanism. ${ }^{10}$ It is known, however, that distal axonal atrophy can lead to secondary demyelination in the presence of healthy Schwann cells. ${ }^{211}$ More recently it has been shown that a small amount of damage to Schwann cells by a demyelinating agent markedly increases the amount of demyelination associated with atrophic axons. ${ }^{12}$ These results, together with the present findings, support the possibility that a "double-crush" or (more correctly) a "double-entrapment" mechanism could operate in certain types of human neuropathy.

We thank Mrs A-M Jones for technical assistance. We are also grateful to Dr P M Le Quesne and Professor P K Thomas for helpful comments and criticism. KR was supported by the Wellcome Trust and JH O'N by the National Fund for Research into Crippling Diseases.

\section{References}

1 Baba M, Fowler CJ, Jacobs JM, Gilliatt RW. Changes in peripheral nerve fibres distal to a constriction. $J \mathrm{Neu}$ rol Sci 1982;54:197-208.

2 Baba M, Gilliatt RW, Jacobs JM. Recovery of distal changes after nerve constriction by a ligature. $J$ Neurol Sci 1983;60:235-46.

3 Morgan-Hughes JA, Sinclair S, Durston JHJ. The pattern of peripheral nerve regeneration induced by crush in rats with severe acrylamide neuropathy. Brain 1974;97:235-50.

4 Krarup C, Gilliatt RW. Some effects of prolonged constriction on nerve regeneration in the rabbit. $J$ Neurol Sci 1985;68:1-14.

5 Hoffman PN, Griffin JW, Price DL. Control of axonal caliber by neurofilament transport. $J$ Cell Biol 1984;99:705-14.

6 Shiraishi S, Le Quesne PM, Gajree T. The effect of vincristine on nerve regeneration in the rat: an electrophysiological study. J Neurol Sci 1985;71:9-17. 
7 Cavanagh JB, Gysbers MF. "Dying back" above a nerve ligature produced by acrylamide. Acta Neuropathol (Berl) 1980;51:169-77.

8 Upton ARM, McComas AJ. The double crush in nerveentrapment syndromes. Lancet 1973;ii:359-61.

9 Williams IR, Gilliatt RW. Regeneration distal to a prolonged conduction block. J Neurol Sci 1977;33:267-73.

10 Frith RW, Litchy WJ. Electrophysiologic abnormalities of peripheral nerves in patients with cervical radiculopathy. Muscle Nerve 1985;8:613.

11 Dyck PJ, Lais AC, Karnes JL, et al. Permanent axotomy, a model of axonal atrophy and secondary demyelination and remyelination. Ann Neurol 1981;9:575-83.

12 Baba M, Gilliatt RW, Harding AE, Reiners K. Demyelination following diphtheria toxin in the presence of axonal atrophy. J Neurol Sci 1984;64:199-211. 